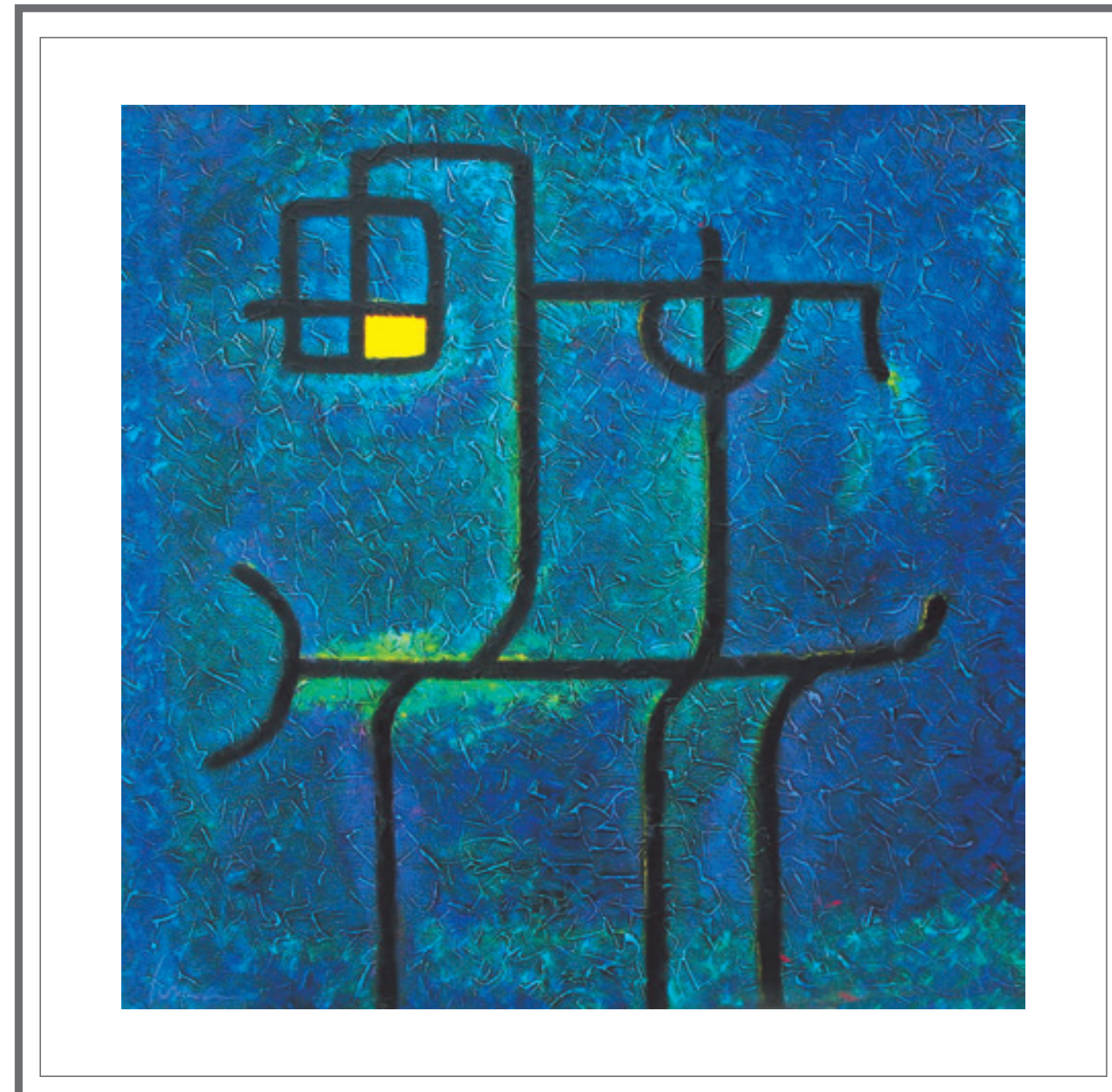

Pintura

Títulos:

Perro Ladrando (con ventana encendida por si las moscas) 


\section{Autor}

Luis Fernando Luna

Técnica:

Acrílico sobre tela

$100 \mathrm{~cm} \times 100 \mathrm{~cm}$ 


\title{
ALGUNOS FACTORES QUE AFECTARON EL SALARIO DE LA MUJER MEXICANA EN LA APERTURA COMERCIAL
}

\author{
SOME FACTORS THAT AFFECTED THE WAGE OF MEXICAN \\ WOMEN AFTER THE FREE TRADE SITUATION
}

José Raúl Luyando Cuevas ${ }^{1}$

\begin{abstract}
Resumen
El mercado asalariado de la mujer en México ha sufrido grandes cambios. Por un lado, hay una creciente a incorporación de la mujer a éste y, por otro, podemos observar un constante crecimiento en sus niveles escolares promedio. Por tanto, el presente trabajo se avoca a investigar los cambios salariales de estas mujeres, antes y después de la apertura comercial mexicana.
\end{abstract}

\section{Palabras Clave}

Salario, Mujer, Apertura comercial

\begin{abstract}
The market wage for women in Mexico has undergone major changes. On one hand, there is a growing incorporation of women into this and, secondly, we see a steady growth in average educational levels.

Therefore, the present study is directed to investigate the wage changes of these women before and after the commercial opening in Mexico.
\end{abstract}

\section{Key words}

Wage, woman, commercial opening

1 Doctor en economía. Profesor-investigador en la Universidad Autónoma de Nuevo León, Instituto de Investigaciones Sociales, México. 


\section{Introducción}

En la década de los ochenta se dieron grandes cambios salariales tanto en hombres como en mujeres. Un rasgo que caracterizó este cambio fue la escolaridad que pasó a ser uno de los principales factores que afecta los cambios salariales. En efecto, uno de los fenómenos más estudiado en los años ochenta y noventa en los Estados Unidos de Norteamérica (E.U.), fue el cambio que sufrió la estructura salarial en los trabajadores. Desde los años setenta su mercado laboral se caracterizó por tres hechos importantes, una marcada desigualdad en la distribución de los ingresos, un lento crecimiento de los mismos y un lento crecimiento en la productividad de los trabajadores. Autores como Kevin Murphy y Finish Whelch (1991); McKinley et al (1990/91), Marvin Kosters (1989), Juhn, Kevin Murphy y Brooks Pierce (1993) y muchos más, han documentado ampliamente estos hechos. Cuando ellos y hablan de una desigualdad en la distribución de los ingresos de los trabajadores, en general, hablan de un hecho que fue consolidándose: un marcado aumento en los sueldos y salarios de los trabajadores con un mayor nivel de escolaridad, en el sentido que ello influye en la productividad del trabajador y en su cualificación ${ }^{2}$. El hecho anterior tiene un amplio consenso, pero no así el fenómeno que lo produce. Se han tratado de dar muchas explicaciones, pero una que es muy destacada y que ha captado el interés de numerosos investigadores en todo el mundo, es que este fenómeno está relacionado a la creciente apertura comercial de los E.U. y al creciente avance de la tecnología.

Como era de esperarse, este fenómeno también afectó al mercado laboral mexicano y la mayoría de las investigaciones que se han hecho para este país, se hacen desde la perspectiva antes planteada, es decir: ¿Cómo la apertura comercial y los cambios tecnológicos han afectado el salario de los trabajadores? Recordemos que México inicia su apertura comercial precisamente en los años ochenta: en 1985, México ingresa al Acuerdo General Sobre Aranceles Aduaneros y Comercio (GATT), donde el gobierno mexicano se compromete a rebajar los aranceles y a eliminar barreras no arancelarias ${ }^{3}$ - se termina así con cuatro décadas de industrialización, mediante el modelo de sustitución de importaciones-. La idea era definir una nueva política económica, caracterizada por la creciente importancia del sector privado en los procesos económicos, al someter el aparato productivo nacional a una fuerte presión para elevar sus niveles de eficiencia y productividad. En este sentido, el objetivo era

2 El concepto de cualificación es utilizado, entre otros en el sistema mexicano de forma similar al concepto de titulación empleado en el sistema del Reino Unido y recogido por el SENA bajo la siguiente definición: una Titulación Laboral es el conjunto de Normas de Competencia Laboral que describe los desempeños requeridos en un Campo Ocupacional o en una Ocupación y que los trabajadores ejercen en varios puestos de trabajo del proceso productivo con tecnologías afines y complementarias, comparen un entorno laboral similar, aplican principios y conocimientos comunes y entregan productos similares en la cadena de valor del sistema productivo.

3 El gobierno mexicano protegió, durante décadas, a los artículos producidos al interior de su economía mediante el uso de tarifas y permisos de importación. Las tarifas incrementaban el precio de importación de los artículos, mientras que los permisos limitaban el número de artículos importados. 
impulsar la exportación competitiva de manufacturas en el mercado internacional. En 1989, el gobierno mexicano modera las restricciones para extranjeros sobre posesión de activos en territorio nacional ${ }^{4}$. En los años noventa la apertura continúa y el primero de enero de 1994 entra en vigor el Tratado de Libre Comercio para América del Norte (TLC), suscrito por los Jefes de Estado de México, Canadá y Los Estados Unidos de Norteamérica y ratificado por los órganos legislativos de los tres países. Con este tratado se consolidan y extienden las reformas de 1985 y 1989.

Las diferentes investigaciones sobre este tema, en general, se centraron en el mercado de trabajo en su totalidad, apoyándose en diferentes variables relacionadas con el cambio tecnológico o a la apertura comercial. Una apuesta que hacen los países no desarrollados con la apertura comercial es que ésta generará una mayor eficiencia en los procesos productivos internos. Por una parte, las empresas domésticas se verán en la necesidad de importar nuevas tecnologías, para poder modernizar sus procesos productivos, y de esta manera, competir por las ganancias en este nuevo contexto internacional. Por otra parte, al establecer mejores condiciones para la entrada de inversión extranjera destinada al sector productivo del país, traería consigo algunos de los procesos productivos más recientes a nivel mundial, dado que buscaría obtener las mayores ganancias posibles. En general y sin entrar en detalles (que hay muchos), se puede ligar la apertura comercial y el desarrollo tecnológico en un país como México ${ }^{5}$. La lógica en este argumento, es que el país venía de un modelo de sustitución de importaciones, un modelo que sirvió en un período determinado, pero, al no darle un seguimiento en sus resultados, terminó por ser un lastre para los procesos productivos del país ${ }^{6}$. Al abrirse la economía, las empresas domésticas requerían de procesos productivos con los que pudieran competir en costos con las empresas mundiales y esto sólo podía darse mediante la importación de estos procesos y la capacitación de sus trabajadores?

4 "Como es del dominio público, el gobierno de De la Madrid ha enfatizado en varias ocasiones su deseo de fomentar la llegada de nueva inversión extranjera directa ofreciendo aplicar con mucha flexibilidad, en un tratamiento caso por caso, las leyes mexicanas restrictivas sobre inversión extranjera y transferencia de tecnología expedidas en el sexenio de Echeverría. En este sentido, a inicios de 1984 el gobierno expidió nuevos lineamientos que especifican las condiciones en las cuales se puede autorizar la propiedad extranjera mayoritaria tanto en empresas nuevas como en las que ya están operando. A este respecto, se considera la posibilidad de otorgar exenciones en industrias que sustituyan importaciones clave, en industrias con alto potencial de exportación, en actividades intensivas en mano de obra y en algunas otras actividades específicas" Guillén, Héctor (1988: p. 54)

5 Para una descripción más detallada, se puede consultar el trabajo de investigación posdoctoral intitulado: "Factores que han Influido en la Determinación de la Tasa Salarial en el Mercado de Trabajo Mexicano en Cuatro Periodos: 1984 (Antes de la Apertura Comercial), 1992 (Con el Ingreso de México al GATT), 2000 (Con el Ingreso de México al TLC) y 2006 (A 21 años de que Ingresó México al GATT y a 12 de que Ingresó al TLC)" en el posgrado de Estudios Sociales de la Universidad Autónoma Metropolitana Iztapalapa.

6 Empresas a las que no importaba modernizar sus procesos productivos, dado que tenían aseguradas sus ganancias -al estar restringida la competencia- y trabajadores anquilosados por la misma situación, en ocupaciones que se tornaron repetitivas. Es decir, empresarios ricos, con empresas pobres, en donde se invertía sólo lo suficiente para mantener la misma situación productiva, y en donde, el resto de las ganancias obtenidas eran utilizadas para realizar consumo superfluo.

7 Los nuevos procesos productivos se tenían que importar, dado que al haber una protección férrea del Estado a la competencia externa en el modelo de sustitución de importaciones, los procesos productivos utilizados hasta antes de la apertura comercial, eran en general, procesos productivos atrasados. 
En nuestro caso, la idea es investigar los cambios salariales que acontecieron en el mercado salarial de la mujer en México, comparando los salarios y características de las asalariadas (principalmente las escolares), antes de la apertura comercial y lo que podemos denominar, la primera etapa de la apertura comercial (1992, cuando México ya había ingresado al GATT), la segunda etapa (2000, cuando México ya había ingresado al TLC) y la tercera (2006, cuando pensamos que la apertura ya se encontraba en una etapa de consolidación). Se supone que hay un cambio tecnológico en los procesos productivos de algunas de las empresas domésticas y otras que vinieron a instalarse desde el exterior y que requieren de trabajadores con mayores cualificaciones.

\section{Algunos Aspectos del Mercado de Trabajo de la Mujer Asalariada}

La incorporación de la mujer al ámbito laboral ha experimentado un rápido crecimiento en todo el mundo. Las causas de este fenómeno por lo regular están ubicadas en dos vertientes: el aumento que ha experimentado la formación escolar de la mujer y la preocupación de muchas legislaturas por combatir el trato desigual entre ciudadanos. México no podía estar al margen de esta situación y a partir de los años que se estudian en este documento podemos verificar que la incorporación de la mujer al mercado laboral mexicano es cada vez mayor, como se muestra en la siguiente Gráfica 1.

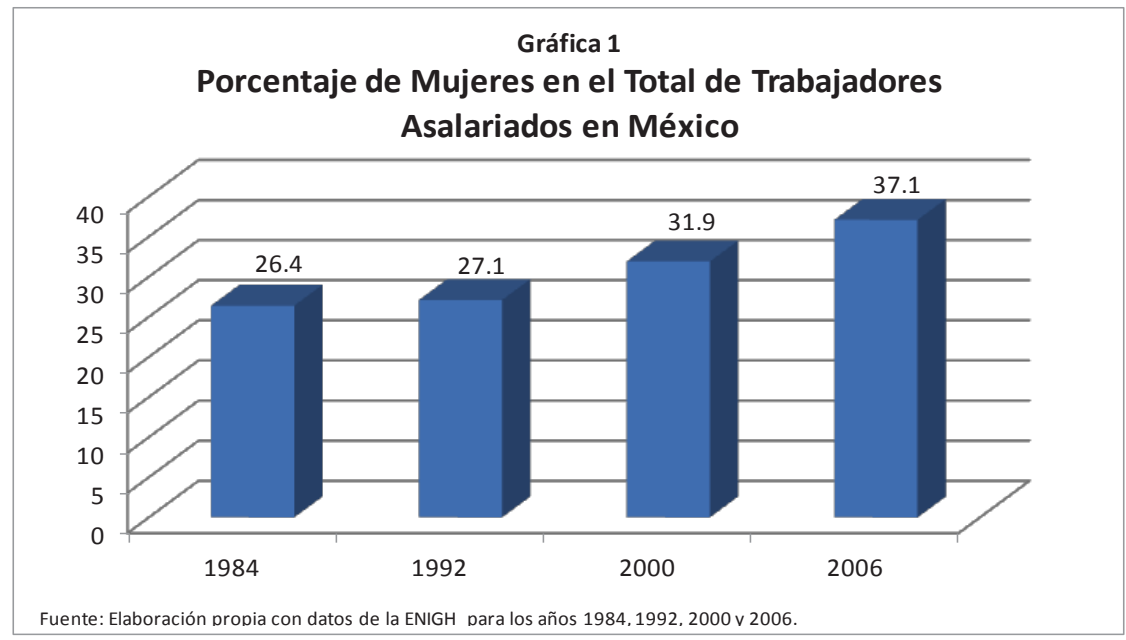


En el caso de México, en cuanto al combate en el trato desigual en el mercado laboral, este queda establecido en su Constitución y en la Ley Federal del Trabajo. En la Constitución Política de los Estados Unidos Mexicanos, en su artículo quinto, queda establecido que a ninguna persona se le podrá impedir pueda dedicarse a la profesión, industria, comercio o trabajo que le acomode, siendo lícitos y en su artículo 123, que para trabajo igual debe corresponder salario igual, sin tener en cuenta el sexo, ni la nacionalidad. Y en la ley federal del trabajo, en su artículo 164, queda establecido que las mujeres disfrutan de los mismos derechos y tienen las mismas obligaciones que los hombres.

En lo referente a la evolución de la formación escolar de la mujer en México se puede señalar que para el tiempo de estudio que considera esta investigación, su progresión ha sido notable. Para el año de 1984, el nivel promedio de escolaridad que alcanzan las trabajadoras asalariadas era de 7.7 años, para el año de 1992, alcanzaron una escolaridad promedio de 8.3, para el año 2000, aumenta a 9.4 y para el año 2006, alcanza un promedio de $9.9^{8}$. Un promedio mayor cada año, al encontrado para la totalidad de los trabajadores asalariados en México.

En las Gráficas 2, 3, 4 y 5 se puede observar el comportamiento de la escolaridad para mujeres asalariadas en los cuatro años.

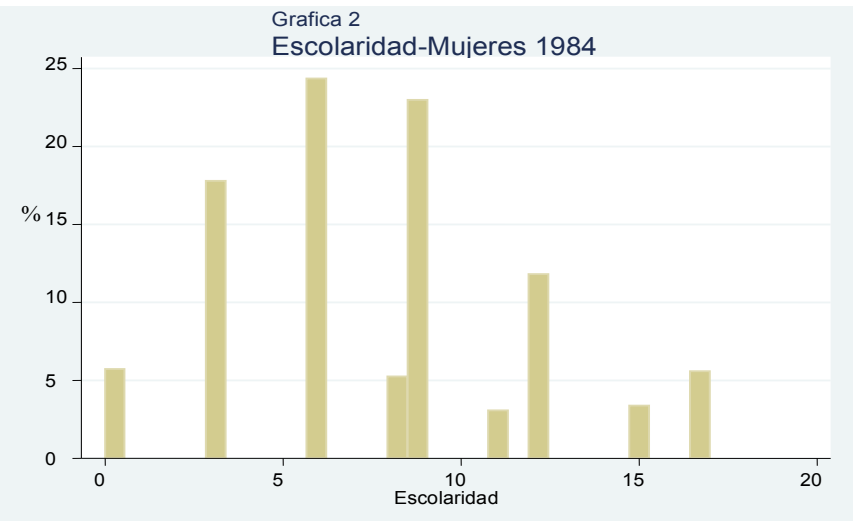

8 Los promedios y los porcentajes se obtuvieron de las bases de datos de la Encuesta Nacional de Ingreso Gasto de los Hogares (ENIGH), para trabajadores asalariados de los años 1984, 1992, 2000 y 2006. Cabe señalar que el nivel de instrucción se codificó de la siguiente manera: 0 para trabajadoras que no tenían ninguna instrucción, contaban con instrucción preescolar o cursaron el primero de primaria, sin haberlo concluido. El 3 es para las trabajadoras con primaria incompleta (los que no obtuvieron su certificado). El 6 para quienes terminaron los estudios de primaria. El 8 para los trabajadoras con secundaria inconclusa. El 9 para los trabajadoras con secundaria conclusa. El 11 para trabajadoras con estudios preuniversitarios inconclusos. El 12 para trabajadoras con estudios preuniversitarios conclusos. El 15 para trabajadoras con estudios universitarios inconclusos. El 17 para trabajadoras con estudios universitarios concluidos. El 18 para trabajadoras con estudios de posgrado. 

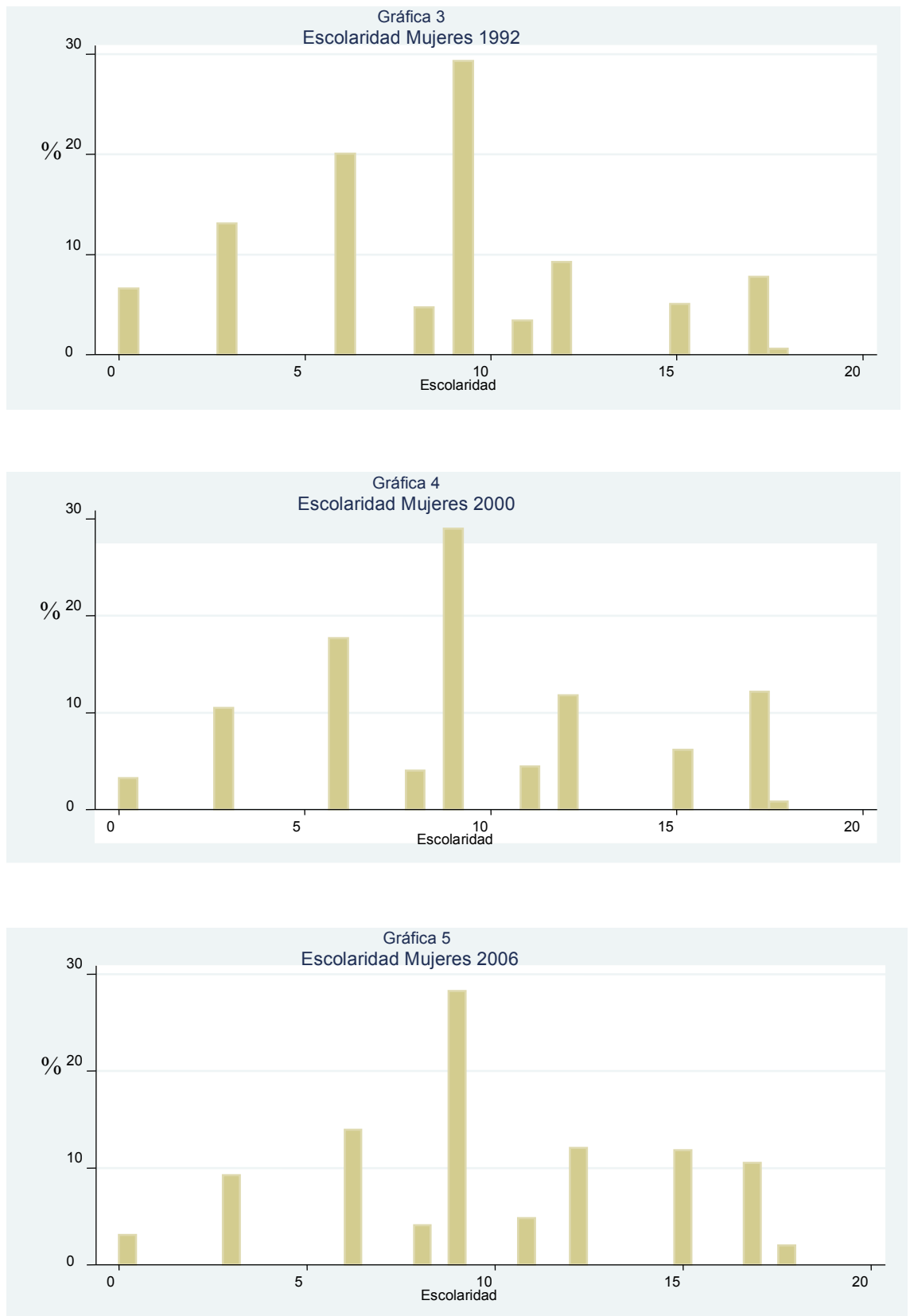

Fuente: Elaboración propia con datos de las ENIGH para los años 1984, 1992, 2000 y 2006 
En las anteriores Gráficas se puede observar claramente cómo la escolaridad de la mujer tiene una tendencia creciente, es decir, el nivel escolar de la mujer va en aumento y cada vez hay un mayor porcentaje de ellas situado en la educación superior a la básica. Para 1984, el mayor porcentaje de ellas se encontraba distribuido en la escolaridad básica. Pero lo anterior cambia en los periodos de estudio (1992, 2000 y 2006), la escolaridad de la mujer se incrementa conforme se pasa de un periodo anterior a uno posterior en forma constante y ahora un mayor porcentaje de ellas se ubica en lo que se denomina la educación superior a la básica. ${ }^{9}$

Pero en el caso de México, y quizá algunos otros países, parece que la incorporación puede tener un tercer elemento, el factor económico, es decir, la mujer se ha visto obligada a integrarse al mercado laboral ante la caída en el poder adquisitivo de los salarios en México, para, de esta manera, aportar otro salario que pueda completar el gasto requerido por las familias.

La caída en el poder adquisitivo de los salarios, la podemos observar mediante la caída en términos reales del salario mínimo en México (un referente primordial en las negociaciones salariales), como se muestra en la Gráfica 6.

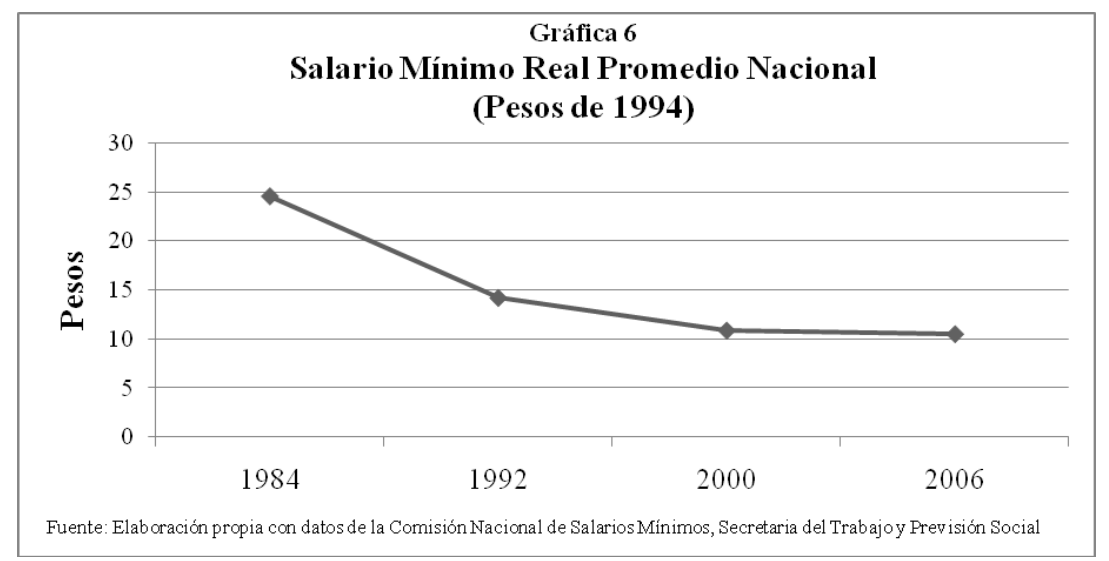

Además del anterior indicador que resulta muy elocuente, se puede señalar que el porcentaje de esposas trabajadoras aumenta de 1984 al año 2006, al pasar de ser $26.7 \%$ en 1984 y aumentar a $34.6 \%$ en 2006 . A esto, se puede añadir que también se incrementa el porcentaje de mujeres que son cabeza de hogar, al pasar de $17.4 \%$ a $21.8 \%$, para los mismos años.

9 En México se considera como educación básica a la que alcanza la terminación de la escolaridad denominada secundaria (aunque para 1984 sólo se consideraba la educación denominada primaria). Por tanto, la educación superior a la básica, es aquella por arriba del nivel denominado secundario terminado ( 9 en la Gráfica). 
Para tener un panorama más amplio de este fenómeno, se muestran las Gráficas de los salarios nominales de las mujeres (salarios mes pasado (SMP)) mediante una distribución absoluta de frecuencias para cada uno de los años estudiados:
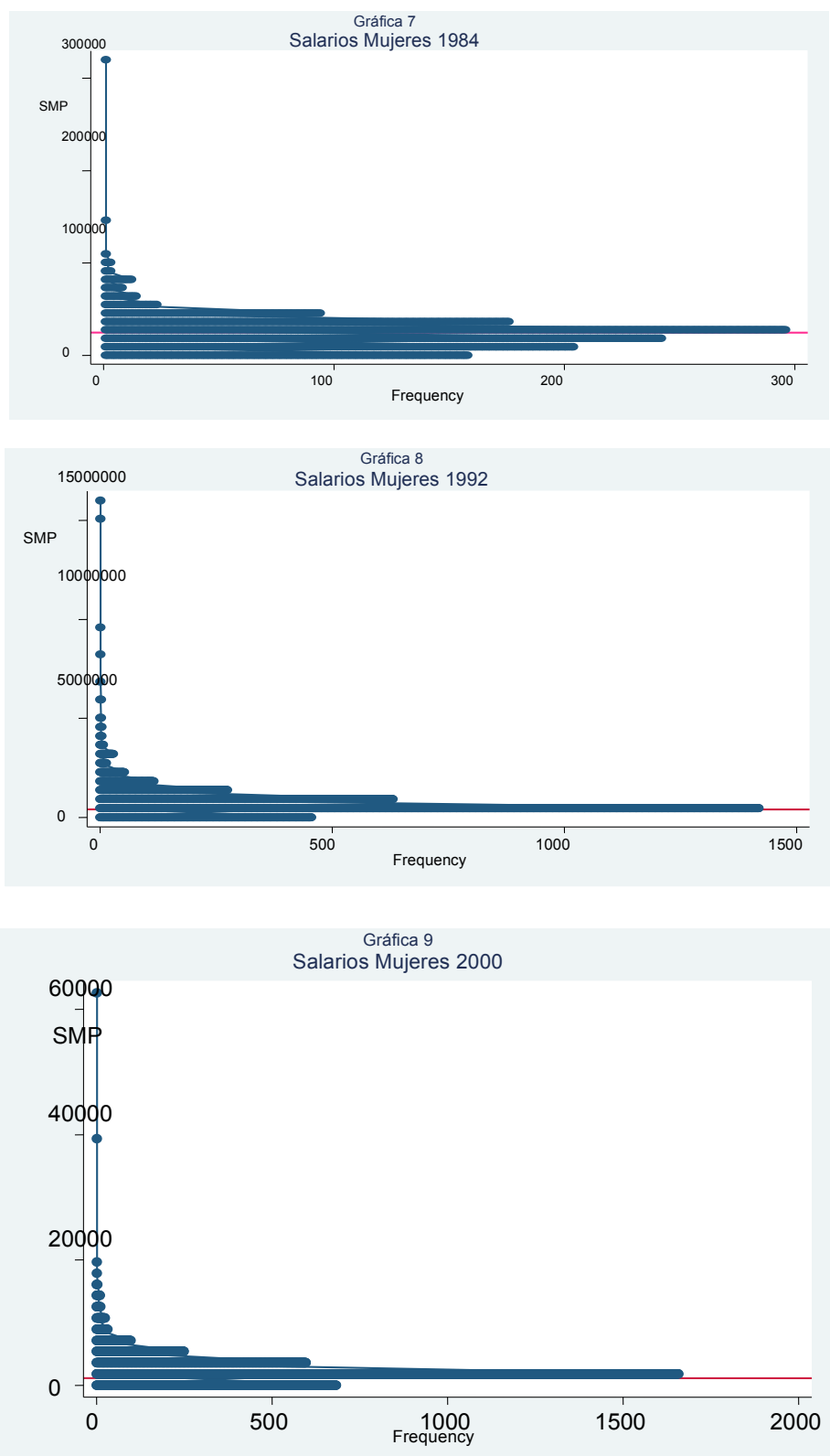


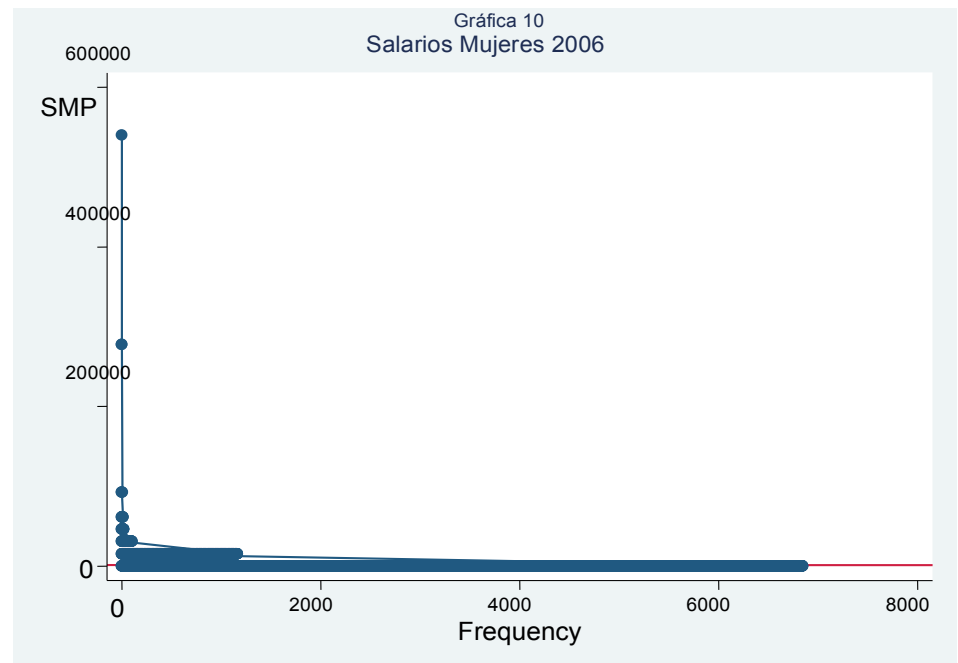

Fuente: Elaboración propia con datos de la ENIGH para los años 1984, 1992, 2000 y 2006

En estas Gráficas se aprecia la evolución en la distribución de los salarios entre las mujeres. En la Gráfica de 1984, los salarios se encuentran concentrados alrededor del salario mínimo (línea horizontal roja), hay pocas mujeres que están por arriba de los diez salarios mínimos, pero hay un buen porcentaje de ellas por arriba de los cuatro salarios mínimos. Para 1992, la distribución comienza a alargarse alrededor del salario mínimo, aparecen más mujeres que ganan por arriba de cinco salarios mínimos y también muchas más con más de diez salarios mínimos. Para el año 2000, se da un caso similar al ocurrido en el año de 1992, pero con la salvedad de que ahora hay un número menor de mujeres que obtienen salarios muy altos -en el caso más extremo, el salario es de alrededor de sesenta salarios mínimos-. Para el año 2006, la frecuencia se encuentra concentrada en mayor medida en el salario mínimo, es decir, la mayoría de las mujeres asalariadas ganan un salario mínimo y hay muy pocas que escapan a esta situación. Aunque como ocurrió en 1992 y 2000 existen casos extremos. Pero ahora esa diferencia es más notoria, dado que la mayoría de las mujeres asalariadas obtienen un salario mensual de alrededor de 1,460 pesos y hay mujeres que están por arriba de los 500,000 pesos mensuales. Dado lo anterior, no resulta sorprendente que la desigualdad salarial ha aumentado en la apertura comercial: en 1984 el coeficiente de Gini era 0.358 , para 1992 pasa a 0.409 , para el año 2000 aumenta a 4.18 y para el año 2006 el coeficiente aumenta a 0.483 .

Otro factor que daría indicios de la transformación que sufrió el mercado salarial de la mujer, son los cambios que se operaron en las ocupaciones laborales que 
desempeñaban las mujeres antes y en la apertura comercial. El avance en el nivel de estudios, según nuestra hipótesis inicial, nos debería indicar que la cualificación de la mujer ha aumentado y ello se debería reflejar en el tipo ocupaciones laborales que desempeña y en su nivel salarial en la apertura comercial. Para hacerlo, sólo tomamos en cuenta las ocupaciones que en los cuatro años fueron afines.

En la Gráfica 11 se puede observar el cambio en el tipo de ocupaciones que generó la mayor escolaridad en la apertura comercial:

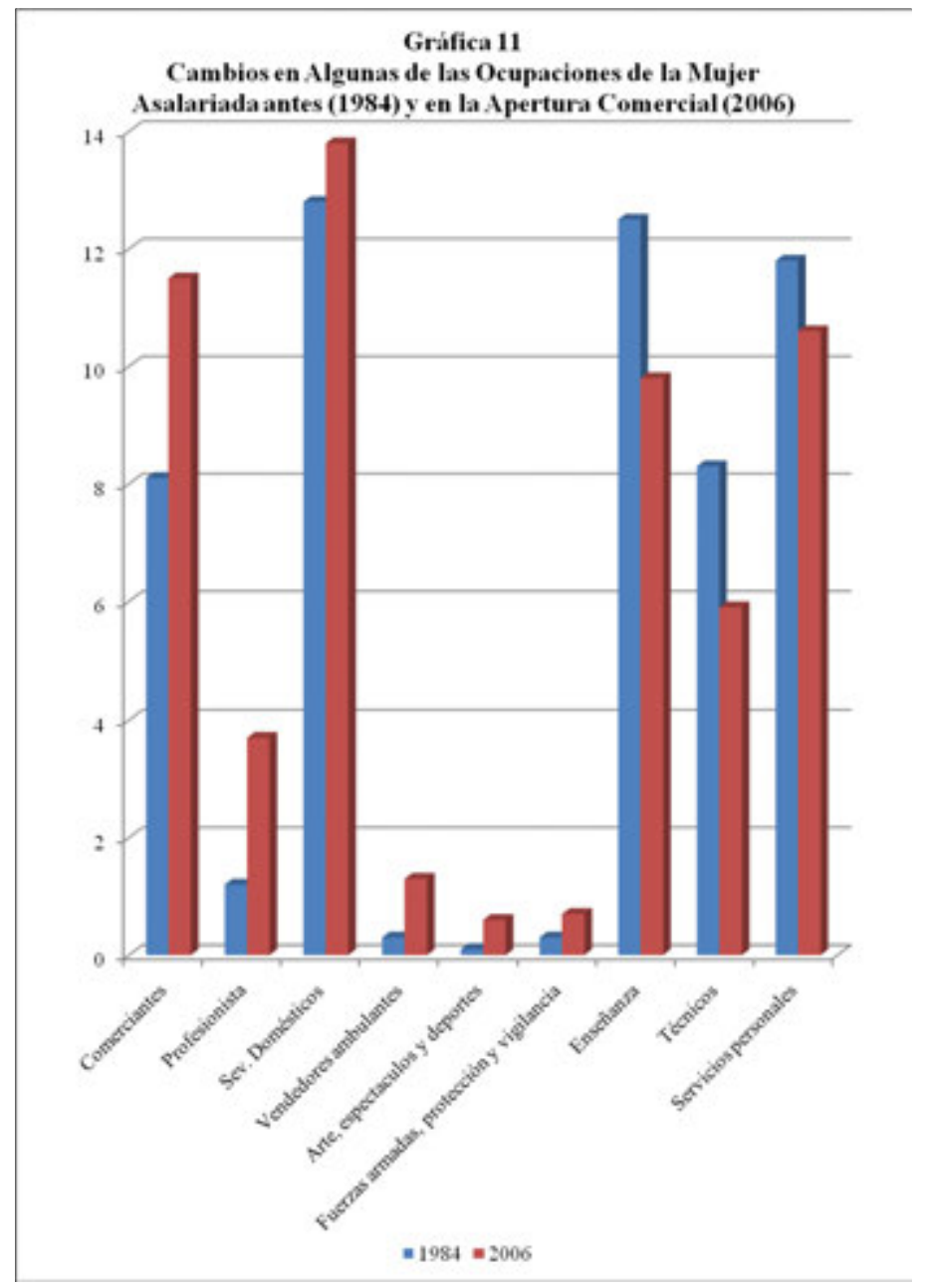

Fuente: Elaboración propia con datos de la ENIGH de los años 1984 y 2006 
Como podría esperarse, con el mayor nivel escolar de las mujeres, la ocupación denominada profesionistas aumentó. Esta situación es interesante pues se hablaría del crecimiento en el número de mujeres con estudios profesionales. Aunque, debemos tener presente que el aumento mayor en términos porcentuales fue en la ocupación de comerciantes, vendedoras y agentes de ventas, donde es conocido que una buena parte de los trabajadores que realizan estas ocupaciones reciben ingresos mediante la modalidad de un pago directo en base al salario mínimo más comisiones, además se dan los casos en que la contratación es de palabra. En tercer lugar aparece la ocupación de servicios domésticos, ocupación que, en términos generales, no requiere de mayores niveles escolares para ser desempeñada eficazmente, con sueldos bajos y en la mayoría de los casos, contratadas mediante acuerdos verbales. En cuarto aparece la ocupación de vendedoras ambulantes, otra ocupación que no requiere de mayores niveles escolares para poderse desarrollar adecuadamente, con sueldos bajos y generalmente con contratos verbales. En quinto lugar aparece la ocupación de arte, espectáculos y deportes, ocupaciones que muchas veces es difícil relacionar a la escolaridad, aunque hay casos en el que el nivel escolar está relacionado a sus sueldos y contrato. Por último, se tiene a la ocupación de fuerzas armadas, protección y vigilancia. En el caso de las fuerzas armadas hay una formación escolarizada al interior de la organización relacionada al salario que obtienen estos trabajadores, pero cabe señalar que es en los rangos menores, donde se concentra la mayoría de estos trabajadores y en estos niveles tan sólo requiere de una formación escolar mínima. En lo referente a protección y vigilancia, hasta fechas recientes se da lo que han dado en llamar la profesionalización de esta ocupación y piden un mayor nivel escolar para ingresar a puestos medios y altos, aunque como en el caso de las fuerzas armadas, la gran cantidad de estas ocupaciones no requiere más que estudios mínimos para ser llevadas a cabo.

Por otra parte, las ocupaciones donde disminuye el porcentaje de mujeres asalariadas es, en primer lugar, el de la enseñanza que requiere últimamente niveles escolares de licenciatura, con contratos generalmente por escrito y sueldos medios. En segundo lugar, la ocupación de técnicos, una ocupación que requiere, en los últimos años, por lo menos de estudios secundarios o preuniversitarios, para posteriormente llevar a cabo los estudios técnicos. En tercer lugar, la ocupación de servicios personales en salones de belleza, lavanderías, baños públicos y los estacionamientos, entre otros, que en la mayoría de los casos requieren de estudios mínimos para ser llevados a cabo, en general, con contratos verbales ${ }^{10}$.

Al tomar estas ocupaciones como parámetro para observar lo que sucede en el mercado laboral, se percata que, aunque aumenta el porcentaje de profesionistas, disminuye el porcentaje de enseñanza y de técnicos y se incrementan los porcentajes

10 La contratación verbal es una contratación que, en general, no otorga las prestaciones que por la ley del trabajo mexicana debería otorgar cualquier patrón. 
de ocupaciones que requieren estudios mínimos como comerciantes, servicios domésticos, vendedores ambulantes y fuerzas armadas, protección y vigilancia. Tal pareciera que avanzan más las ocupaciones que requieren estudios mínimos, pagan salarios bajos y contratan verbalmente. Lo anterior se refuerza, si tomamos en cuenta que en 1984, el porcentaje de trabajadoras con contrato por escrito era de $64 \%$ y en el año 2006 pasa a 55.7\%, es decir, aumenta el número de mujeres contratadas mediante convenio verbal. También, disminuye el número de mujeres afiliadas a algún sindicato, pues en 1984, el porcentaje de mujeres sindicalizadas era de $31.8 \%$ y pasa a ser de $19.7 \%$ en el año 2006 , esto nos dejaría ver que además de no tener contrato por escrito, pierden su poder de negociar sus salarios colectivamente y lo tienen que hacer individualmente, con lo que significa esto último en un país donde los sueldos reales han caído en más de la mitad en el periodo estudiado.

\section{La Relación Escolaridad-Salario}

Del panorama anterior queda la sensación de que la relación positiva entre escolaridad y salario se ha perdido en la apertura comercial. Para tener un panorama más adecuado de esta relación, se hace la Gráfica 12, para los cuatro años.

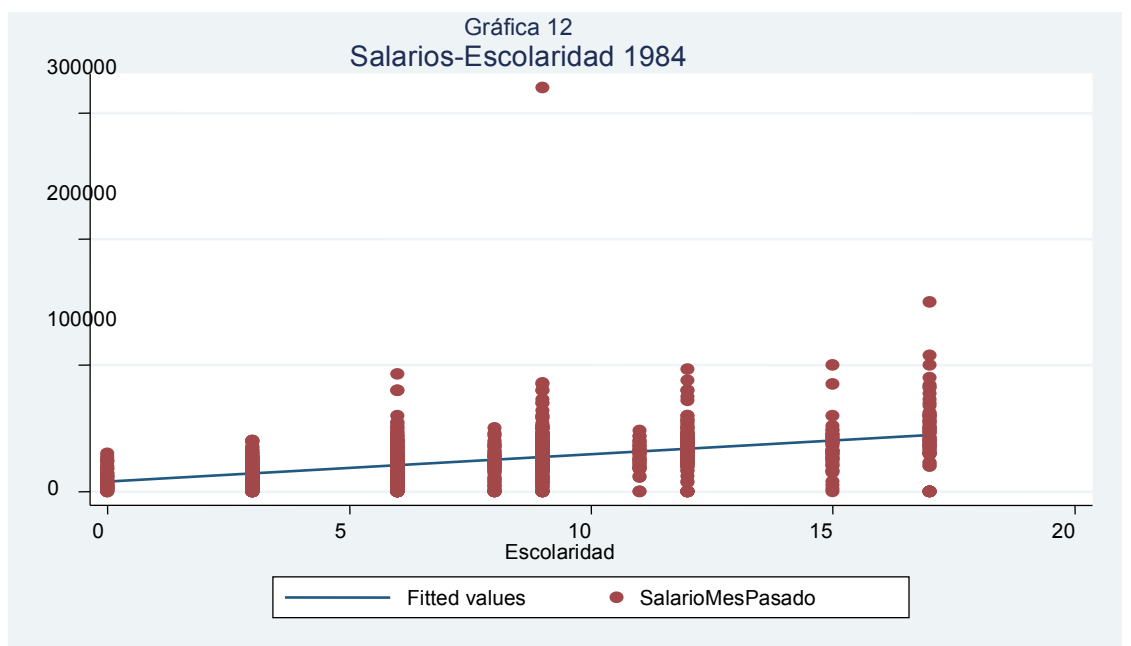

Fuente: Elaboración propia con datos de la ENIGH del año 1984 
Antes de la apertura comercial, se puede apreciar una relación positiva entre la escolaridad y el salario de las mujeres. Una relación que sólo es distorsionada por uno de los puntos. Este punto indica que una de las mujeres con estudios secundarios obtiene un salario muy elevado. Fuera del punto anterior podemos observar, en general, una tendencia creciente positiva entre escolaridad y salario de la mujer. Para apreciar de un modo más claro esta relación se hace un ajuste lineal -para ver la tendencia lineal promedio de los datos, línea negra-. El ajuste indica una relación lineal promedio creciente. Es decir, en promedio a mayores estudios mayor nivel salarial.

Ahora veamos lo que ocurre en la primera parte de la apertura comercial con la relación encontrada anteriormente. A continuación podemos observar la Gráfica 13 para el año de 1992.

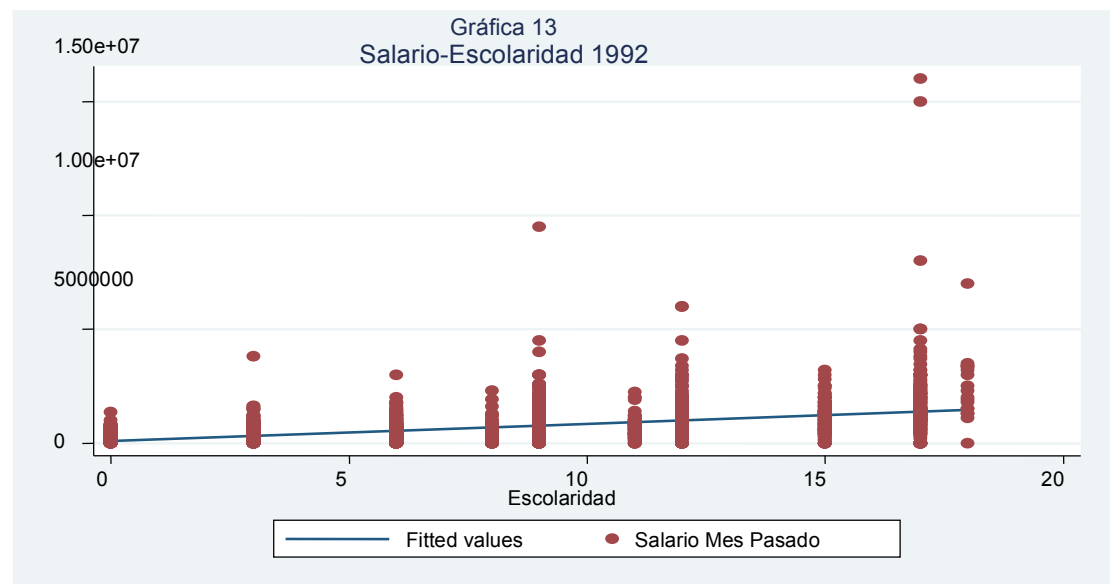

Fuente: Elaboración propia con datos de la ENIGH del año 1992

Aunque ahora los puntos extremos los encontramos en mujeres con estudios universitarios concluidos, podemos apreciar que en promedio la relación lineal entre escolaridad y salario de las mujeres es menor a la observada en 1984. La relación positiva encontrada en el año anterior aún existe, pero ahora podemos observar que es menor a la existente antes de la apertura comercial. 
En la Gráfica 14 lo que ocurrió en el año 2000:

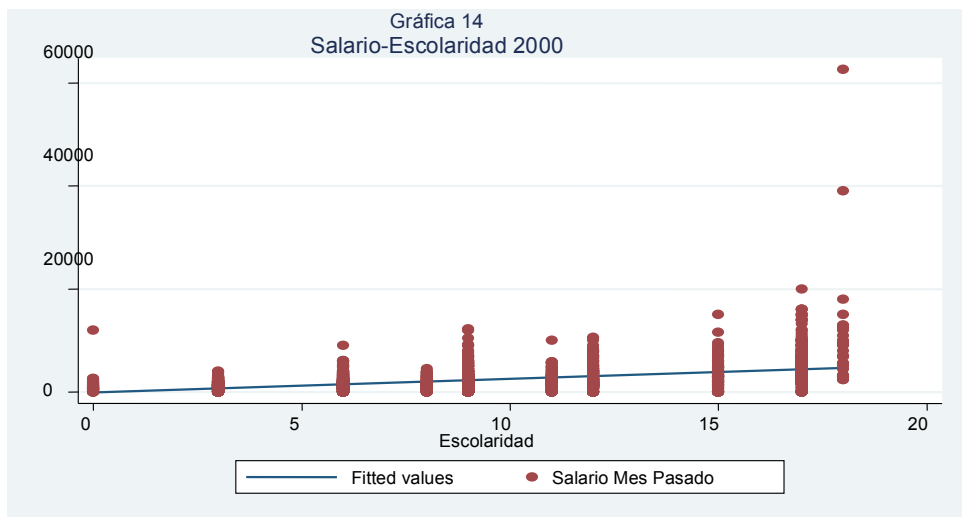

Fuente: Elaboración propia con datos de la ENIGH de año 2000.

En el Gráfico 14 se aprecia que ahora los puntos extremos se ubican en mujeres con estudios de posgrado. Lo anterior podría parecer una buena noticia, pues estos puntos podrían sesgar el resultado promedio al hacer que la línea de ajuste lineal tuviera una pendiente positiva mayor. Pero, contrario a esto, la relación positiva disminuye con respecto a la que había en los años anteriores. Es decir, la relación promedio entre escolaridad y salario disminuye. Aunque la escolaridad es un factor que afecta positivamente el salario de las mujeres, su efecto en la apertura comercial disminuye.

Lo que ocurre en el año 2006, está en la Gráfica 15.

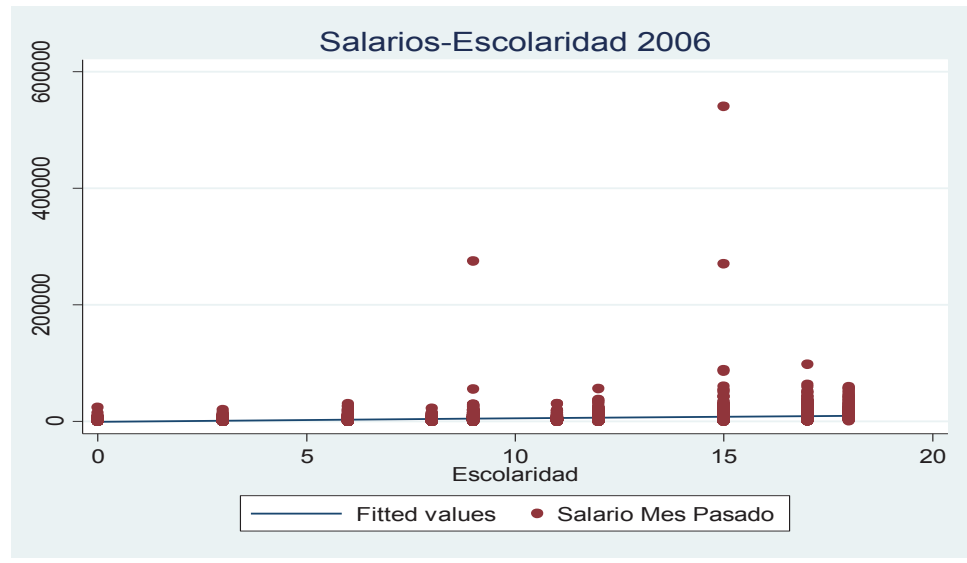

Fuente: Elaboración propia con datos de la ENIGH del año 2006. 
Los puntos extremos ahora los encontramos en mujeres con escolaridad universitaria inconclusa y con secundaria terminada. Lo anterior podría ser un indicio de la pérdida de la escolaridad como uno de los factores determinantes en el salario de las mujeres. La línea de ajuste lineal, nos mostraría que la pendiente de la misma disminuye. Es decir, en promedio la relación entre escolaridad y salarios de las mujeres disminuye con respecto a la que había en los años anteriores, el efecto de la escolaridad sobre el salario de las mujeres disminuye conforme avanza la apertura comercial.

\section{Conclusiones}

Aunque la apertura comercial es un parámetro que se toma para observar la relación entre escolaridad y salario, no se le puede atribuir este efecto en un grado específico a ella, dado que en ningún sentido el trabajo ha medido este fenómeno. Lo que sí se puede señalar es que la relación entre escolaridad y salario pierde importancia conforme avanza la apertura comercial. Mas precisamente, tenemos indicios que la relación entre salarios y escolaridad en promedio ha perdido importancia para la mujer en la apertura comercial. La escolaridad disminuye su importancia como uno de los factores que afectan el salario de las mujeres y como factor de movilidad social. La escolaridad en este sentido, pierde una de las razones que le dan significado en toda sociedad, y por tanto, hay otros factores que pasan a ocupar el lugar que ésta tenía antes. Algunos de ellos, en una sociedad como la mexicana, con sus grandes desigualdades sociales, son menos democráticos que la escolaridad y, muchas veces, pasan a depender más de las redes sociales con que cuentan las mujeres y sus familias, que de las capacidades y cualificaciones que en un momento dado se le pueden atribuir a la formación escolar ${ }^{11}$. La situación anterior en parte podría explicar algunas de las situaciones encontradas en la investigación, es decir, que personas con bajos niveles escolares ocupen puestos donde obtienen altos salarios (muy superiores a los obtenidos por el resto de las mujeres) ${ }^{12}$.

Por otra parte, la política implementada por el gobierno mexicano, mediante el denominado Pacto de Solidaridad Económica ${ }^{13}$, para atar el incremento de los salarios

11 La información que se puede obtener de las condiciones del mercado laboral y de propuestas de trabajo que pueden cubrir, aún sin tener el perfil o las cualificaciones para ocuparlo, podrían estar más supeditadas a las relaciones o contactos que tienen las mujeres o su familia.

12 En México, esta práctica es muy utilizada en el sector público y en algunos casos en la iniciativa privada. Con el arribo del PAN (Partido Acción Nacional) a la presidencia de México, esta situación se ha acentuado. Con el advenimiento de Felipe Calderón a la presidencia de la República esto ha empeorado, puesto que él mismo ha puesto el ejemplo de poner a sus amigos cercanos e incondicionales en puestos claves. Una práctica que ha permeado a la sociedad en todos sus niveles.

13 Pacto llamado de Solidaridad Económica se firma el 15 de Diciembre de 1987 y sus características principales son: a) El gobierno se compromete a: reducir su gasto programable de 1988 de 22 a $20.5 \%$ con relación al PIB aumentar su superávit primario de 5.4 a $8.3 \%$, también en relación al PIB; suprimir subsidios; reducir más el sector paraestatal y racionalizar sus estructuras administrativas. b) El Congreso del Trabajo declina su reclamo de aumento salarial de emergencia, al aceptar la exhortación al sector privado de aumentar el $15 \%$ de emergencia 
(principalmente el salario mínimo) a la inflación pronosticada por el gobierno en turno, sirvió como un instrumento para bajar los salarios reales de los trabajadores y contener sus demandas. Lo anterior con el propósito de que en la apertura comercial, se pudiera utilizar como un medio para generar la competitividad en costos que requieren las empresas instaladas en el territorio mexicano ante la competencia que tendrían en la apertura comercial con las empresas extranjeras. Es decir, el gobierno mexicano apostó por esta práctica, en lugar de impulsar el desarrollo tecnológico en la producción y de este modo reducir costos por este medio y no sólo mediante mano de obra barata. La situación anterior, influye de manera determinante en los resultados que obtenemos, pues creemos que esta política ha compactado los salarios en el mercado salarial mexicano. Además de que hay algún indicio de que con la misma se ha precarizado el empleo. Es decir, como se vio, la mayoría de los puestos de trabajo creados en este tiempo son de los que pagan bajos salarios, no tienen prestaciones y requieren de trabajadores con escolaridad básica.

La política de los gobiernos mexicanos ha actuado en contra de la postura que indica que a mayor nivel escolar mayor productividad y mayores salarios. Pues, en general, lo que encuentra este trabajo es que aunque los niveles escolares de las mujeres en México han aumentado, ello no se ve reflejado en promedio en sus niveles salariales, ni en los puestos de trabajo promedio que logran obtener. La idea de que la escolaridad sirve como medio de movilidad social y que quizá es el medio más democrático para hacerlo -dado que por precepto constitucional la educación en México es gratuita-, se ha perdido. En la sociedad hay la sensación de que de los estudiantes que obtienen un grado universitario o de posgrado son my pocos los que logran ejercer su profesión y aún menos los que logran obtener un trabajo "bueno" y bien remunerado. En los medios de comunicación mexicanos aparecen reportajes o encuestas de las personas que obtienen un grado universitario o de posgrado y se dedican a oficios que nada tienen que ver con lo que estudian. Es común la persona que con un grado universitario se dedica a ser chofer de taxi, pues sus ingresos son mayores a lo que obtendría en la mayoría de los puestos que ofrece el mercado de trabajo mexicano para un recién egresado. Lo peor de este escenario desde nuestro punto de vista, es que muchos actores sociales, entre ellos los actuales gobiernos panistas, crean la percepción de que no es sólo el nivel escolaridad el que sirve para la movilidad, sino la calidad de esa educación. Pero en un país como México donde se apuesta poco por la investigación, la calidad de la educación -y no la educación -ahora tiene un costo que la mayoría de la población no puede pagar, por tanto, la movilidad va a ser para esos que puedan pagarla o para aquellos que por sus condiciones intelectuales puedan obtenerla por medio de becas u otros apoyos,

a salarios mínimos y contractuales, y el $20 \%$ en enero sólo a los mínimos. Con la promesa que desde marzo se ajustarán mensualmente, de acuerdo a la canasta básica recientemente anunciada.

c. Los campesinos aceptan mantener sus productos a los precios de garantía reales estipulados en 1987.

d. Los empresarios se obligan a aumentar la oferta de productos básicos, a elevar la eficiencia de la planta productiva y a "moderar la variación de todos los precios tomando en cuenta los márgenes de comercialización". 
pues se propaga la noción de que la calidad la pueden ofrecer algunas instituciones de educación privada con prestigio. Esto dice que la concentración de la riqueza se acentuaría aún más, es decir, simple y llanamente que crecería el porcentaje de pobres en el país (que en México según cifras oficiales ronda el 50\%) y se les dejaría sin oportunidades de movilidad social.

Por último, es importante señalar que cuando se firma el Tratado de Libre Comercio para América del Norte, se firma un acuerdo paralelo denominado Acuerdo de Cooperación Laboral para América del Norte (ACLAN), donde se establecen los siguientes principios laborales: 1) Libertad de asociación y protección al derecho a organizarse; 2) Condiciones mínimas de trabajo, como serían el salario mínimo y pago de tiempo extra; 3) Eliminación de la discriminación en el empleo, por causa de raza, sexo religión, edad u otros y 4) Salario igual para hombres y mujeres. Condiciones que como se ha señalado aparecen en las leyes mexicanas y que supondríamos se reforzarían con este acuerdo. Pero la realidad para las mujeres asalariadas en México, parece muy diferente. Su afiliación a sindicatos va en franco decrecimiento. La contratación de palabra o por outsourcing aumenta, y con ello, la pérdida de prestaciones laborales establecidas en las leyes mexicanas y ratificadas en el ACLAN.

\section{ANEXO}

Para obtener los datos empleados en la presente investigación se utilizaron las Encuesta Nacional de Ingreso y Gasto de los Hogares (ENIGH) de los años de 1984, 1992, 2000 y 2006. Con el propósito de crear la base que necesitábamos, utilizamos sólo dos de las bases que se encuentran en la encuesta, la de Ingresos, que detalla el monto de los ingresos y de donde provienen y la llamada Person, donde se detallan las características escolares, sociales y laborales de los miembros del hogar. Estas dos bases se fusionaron en una sola base mediante dos campos comunes, el campo llamado FOLIO, el cual, es un identificador único del hogar y el campo NUMREM, el cual señala el número de renglón del residente del hogar ${ }^{14}$.La intención al fusionarlas, era tener identificadas a las personas que dentro de la familia obtenían algún ingreso, saber cuál era el monto del ingreso, de donde provenía y cuáles eran sus características sociales y laborales.

En los tres años se utiliza lo que se denomina en las encuestas "ingreso mes pasado", es decir, el último ingreso que percibió el trabajador. Se toman sólo los ingresos netos por remuneraciones al salario, es decir, las percepciones que en efectivo

14 Para realizar la fusión se utilizó el programa Acces. Cabe señalar que para los años 1984 y 2000 no hubo mayor problema. Pero en el año de 1992, se tuvo varios problemas que se debieron superar. En primer lugar, hay una buena cantidad de trabajadores que no tenían el campo NUMREM, después de intentar varias soluciones, se decidió dejarlos fuera, por considerar era la solución más indicada. En segundo lugar, varios campos estaban aglutinados en uno solo, por lo que se procedió a dividirlos, por medio de la longitud de cada uno de los campos aglutinados, esto se realizó alprogramar en el mismo Acces. 
obtienen los miembros de hogar a cambio de la venta de su fuerza de trabajo a una empresa, institución o patrón, con quién han establecido determinadas condiciones de trabajo mediante un contrato o acuerdo, en específico hablamos de los sueldos, salarios o jornal y las horas extras

\section{REFERENCIAS BIBLIOGRÁFICAS}

Beth, Mary (2003), Gender and Inequality in the Global Labor Force, Annual Review of Anthropology, Vol. 32, pp. 41-62.

Blackburn, McKinley L., Bloom, David E. and Freeman, Richard, B.(1990/91), An Era of Falling Earning and Rising Inequality, Brookings Review, Winter.

CD-ROM, Encuesta Nacional de Ingreso Gasto de los Hogares (ENIGH), INEGI, México 1984.

CD-ROM, Encuesta Nacional de Ingreso Gasto de los Hogares (ENIGH), INEGI, México1992.

CD-ROM, Encuesta Nacional de Ingreso Gasto de los Hogares (ENIGH), INEGI, México 2000.

CD-ROM, Encuesta Nacional de Ingreso Gasto de los Hogares (ENIGH), INEGI, México 2006.

De la Garza, Enrique (2001), NAFTA, Manufactura y Trabajo, Ponencia Presentada en la XX Asamblea General de CLASCO en la Ciudad de Guadalajara en México, del 21 al 24 de Noviembre.

Gong, Xiaodong and van Sooest, Arthur (2002), Family Structure and Female Labor Supply in Mexico City, The Journal of Human Resources, Vol. 37, $\mathrm{N}^{\circ}$ 1, Winter, pp. 163-191.

Juhn, Chinhui, Murphy, Kevin and Pierce, Books (1993), Wage Inequality and the Rise in Returns to Skill, Economy, number 3, Volume 101, The University of Chicago Press, June.

Kosters, Marving H. (1989), Schooling, Work Experience and Wage Trends, The American Economic Review, Vol.80, N² 2 .

Luyando, José R. (2010), Factores que han Influido en la Determinación de la Tasa Salarial en el Mercado de Trabajo Mexicano en Cuatro Periodos: 1984 (Antes de la Apertura Comercial), 1992 (Con el Ingreso de México al GATT), 2000 (Con el Ingreso de México al TLC) y 2006 (A 21 años de que Ingresó México al GATT y a 12 de que Ingresó al TLC), Investigación Posdoctoral, Posgrado en Estudios Sociales, Universidad Autónoma Metropolitana Iztapalapa, México.

Murphy, Kevin M. And Welch Finis (1991), Wage Premiums for College Graduates: Recent Growth and Possible Explanations, Educational Researcher, May. 